\title{
Intracavernous chondrosarcoma associated with Ollier's disease
}

\author{
D J Brazier, J Roberts-Harry, A Crockard
}

Multiple enchondromatosis (Ollier's disease) ${ }^{1}$ is a rare, non-hereditary condition of unknown aetiology in which cartilaginous masses are found in bones. We report a patient with Ollier's disease who presented with a parasellar syndrome due to a chondrosarcoma in the cavernous sinus. The association of Ollier's disease and intracranial cartilaginous tumours (chondrosarcoma or chondroma) is recognised but very rare.

\section{Case report}

A 36-year-old woman presented with a 4 month history of horizontal diplopia looking to her right. A diagnosis of Ollier's disease had been made in childhood because of skeletal abnormalities. On examination she had $6 / 5$, N5 unaided vision in both eyes. Colour vision was normal to the Ishihara plates. There was a $2 \mathrm{~mm}$ right proptosis and restriction of all movements of the right eye, particularly abduction. Pupils were normal as were facial and corneal sensation. Goldmann fields were normal. There was no abnormality of optic discs or retina on fundus examination. Eye movements were recorded by Hess chart for comparison during follow up.

Systemic examination demonstrated deformity and shortening of the right tibia and fibula, a mass in the right scapula, and gross deformity of both hands. Chest $x$ ray showed enchondromata in the ends of the ribs. Head computed tomography $(\mathrm{CT})$ scan revealed a right parasellar mass (Fig 1) and also showed enchondroma of the ethmoids. She was referred for neurosurgical assessment but, because of the

\section{Department of Ophthalmology, University College Hospital, Gower Street, London WC1E 6AU D J Brazier \\ J Roberts-Harry}

The National Hospital for Neurology and Neurosurgery, Queen Square, London WC1N 3BG

A Crockard

Correspondence to: Mr D John Brazier, University College Hospital, Gower Street, London WCIE 6AU.

Accepted for publication 23 April 1993
Figure 1 Computed tomographic scan showing right orbital fissure. parasellar lesion with smooth outline occluding the superior non-progressive nature of her symptoms, it was decided to await events.

Four months after presentation she developed episodes of severe right frontal pain with vomiting. Right acuity had declined to $6 / 6$ N5 with subjective reduction of light brightness and a right relative afferent pupil defect. The right optic disc remained normal. There was reduced sensation in the territory of the maxillary division of the right trigeminal nerve and less obvious reduction in the cutaneous distribution of the ophthalmic division. Repeat Hess chart suggested that the limitation of right abduction had progressed despite a subjective improvement in diplopia. The degree of proptosis, colour vision, and fields were unaltered. Repeat CT scan did not show any obvious change in the parasellar mass. Carotid angiography showed a relatively avascular lesion in the region of the right cavernous sinus with displacement of the carotid artery anteriorly.

In March 1990, she underwent right pterineal craniotomy and the intracavernous tumour was microscopically completely resected. Recovery from surgery was uneventful. Histological examination (Fig 2) showed a lobulated cartilaginous tumour, most of the cells having compact nuclei, with larger more darkly staining nuclei in some areas. In an adult this appearance was thought to represent a chondrosarcoma of borderline grade I/II malignancy. In a child or young adult in whom the skeleton was still growing the histological diagnosis would have been chondroma.

Most recent follow up was in November 1992, 32 months after surgery. Right visual acuity was $6 / 5$ part, N5. The colour desaturation and afferent pupil defect had resolved and right eye movements had improved. Abduction was increased (with subjective improvement in diplopia on right gaze) and vertical movements had returned to normal on the Hess chart. Some

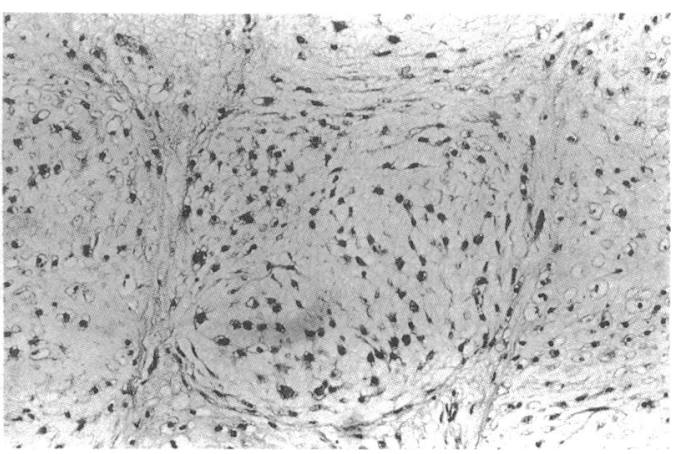

Figure 2 Histology of the parasellar chondrosarcoma. A lobulated cartilaginous tumour with compact nuclei (haematoxylin and eosin). 
restriction of adduction remained but was stable. There was no proptosis with the exophthalmometer and sensation over the right ophthalmic division of the fifth cranial nerve had returned to normal, although a minor reduction in sensation of the maxillary division persisted. Fields and optic discs remained normal.

\section{Comment}

The association of intracranial cartilaginous tumour and Ollier's disease is rare and there are only a handful of similar cases published. Chondromas and chondrosarcomas usually arise from the skull base and are located in the middle cranial fossa. Sarwar' reported a patient with Ollier's disease presenting with diplopia and impaired vision due to chondroma arising from the sphenoid. Horizontal diplopia was also the presenting symptom in the cases reported by Traflet $e t \mathrm{al}^{3}$ and Reuter and Weber. ${ }^{4}$ The patient in the latter report also suffered severe ipsilateral headache associated with nausea and vomiting and paraesthesias in the distribution of the trigeminal nerve. These features are obviously comparable to the case we report. Pospiech and coauthors ${ }^{5}$ reported a sellar chondroma associated with Ollier's disease presenting as a chiasmatic syndrome. This patient had head pain, vomiting, and visual failure.
The main differential diagnosis in the reported case was meningioma although the relatively avascular tumour appearance on carotid angiography was against this. When the tumour contains calcification craniopharyngioma must be considered and when positioned more posteriorly (clivus/cerebellopontine angle) radiological differentiation from chordoma may be difficult. $^{2}$

Our patient was treated by complete surgical tumour removal with no radiological evidence of recurrence to date. Surgery is the mainstay of treatment in these tumours which are not considered chemosensitive or radiosensitive. ${ }^{6}$

We thank Dr Robin Barnard and Dr Tamas Ravesz at the National Hospital for Neurology and Neurosurgery for advice on interpretation of the histology and for preparing the histological pretation of the histology and for preparing the histological
photograph. We also thank Steve Paratian for photographic assistance.

1 Ollier L. De la dyschondroplasie. Bulletin Societe de Chirurgiens de Lyon 1900; 93: 23-4.

2 Sarwar M, Swischuk LE, Schechter MM. Intracranial chondromas. Am $\mathcal{Y}$ Roentgenol 1976; 127: 973-7.

3 Traflet RF, Babaria AR, Barolat G, Doan HT, Gonzalez G, Mishkin MM. Intracranial chondroma in a patient with Ollier's disease. 7 N'eurosurg 1989; 70: 274-6.

+ Reuter K, Weber AL. Parasellar chondrosarcoma in a patient with Ollier's disease. Neuroradiologv 1981; 22: 151-4.

Pospiech J, Mehdorn HM, Reinhardt V, Grote W. Sellar chondroma in a case of Ollier's disease. Neurochirurgica 1989; 32: $30-5$.

6 Souhami R, Tobias J. Cancer and its management. Oxford: Blackwell, 1986: 395-6.

\title{
A rare cause of visual loss in AIDS patients: central retinal vein occlusion
}

\author{
Yahya Ismail, Patrick M Nemechek, Edward L Arsura
}

Department of Medicine, Kern Medical Center, University of California, Los Angeles, School of Medicine, USA Y Ismail* P M Nemechek E L Arsura

$\star$ Dr Ismail is a medical resident in the Department of Medicine, Kern Medical Center/UCLA

†Dr Arsura is associate clinica professor of Medicine at University of California, Los Angeles, School of Medicine. Correspondence to:

Edward L Arsura, MD Department of Medicine, Kern Medical Center, 1830 Flower Street, Bakersfield, CA 93305, USA. Accepted for publication 29 April 1993
Loss of vision in AIDS patients is most commonly associated with cytomegalovirus (CMV) retinitis. ' Central retinal vein occlusion (CRVO) is an uncommon condition which has been reported once previously to cause visual loss in a

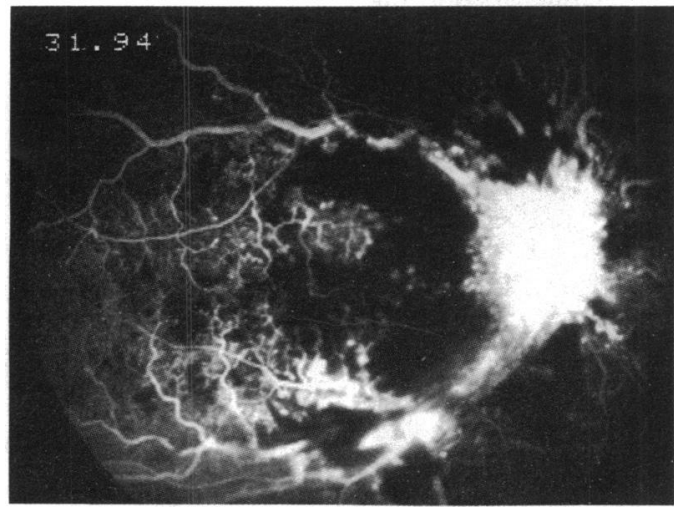

Figure 1 Fluorescein angiography of the right eye showing extensive retinal haemorrhages, blurred disc margins, and dilated veins. patient with AIDS. ${ }^{2}$ In this report we describe an additional case of CRVO in an AIDS patient who presented with acute loss of vision temporally related to treatment of anaemia with recombinant human erythropoietin (r-HuEPO).

\section{Case report}

A 31-year-old woman first tested positive for HIV infection in 1986. She acquired the infection during a 9 year period of intravenous drug use. Her illness had been complicated by multiple hospital admissions for Pneumocystis carinit pneumonia, Staphylococcus aureus pneumonia, and septicaemia. At the time of presentation her medications included zidovudine, fluconazole, acyclovir, dapsone, imipramine, clonazepam, and r-HuEPO. r-HuEPO had been started 2 weeks before CRVO for anaemia associated with zidovudine treatment.

At presentation she complained of sudden, painless loss of vision in the right eye. The visual examination showed significant diminution of 